\title{
Crossings and Nestings for Arc-Coloured Permutations
}

\section{Lily Yen}

Dept. of Math. \& Stats., Capilano University, North Vancouver, B.C., Canada; also: Dept. of Math., Simon Fraser University, Burnaby, B.C., Canada

\begin{abstract}
The equidistribution of many crossing and nesting statistics exists in several combinatorial objects like matchings, set partitions, permutations, and embedded labelled graphs. The involutions switching nesting and crossing numbers for set partitions given by Krattenthaler, also by Chen, Deng, Du, Stanley, and Yan, and for permutations given by Burrill, Mishna, and Post involved passing through tableau-like objects. Recently, Chen and Guo for matchings, and Marberg for set partitions extended the result to coloured arc annotated diagrams. We prove that symmetric joint distribution continues to hold for arc-coloured permutations. As in Marberg's recent work, but through a different interpretation, we also conclude that the ordinary generating functions for all $j$-noncrossing, $k$-nonnesting, $r$-coloured permutations according to size $n$ are rational functions. We use the interpretation to automate the generation of these rational series for both noncrossing and nonnesting coloured set partitions and permutations.

L'équidistribution de plusieurs statistiques décrites en termes d'emboitements et de chevauchements d'arcs s'observes dans plusieurs familles d'objects combinatoires, tels que les couplages, partitions d'ensembles, permutations et graphes étiquetés. L'involution échangeant le nombre d'emboitements et de chevauchements dans les partitions d'ensemble due à Krattenthaler, et aussi Chen, Deng, Du, Stanley et Yan, et l'involution similaire dans les permutations due à Burrill, Mishna et Post, requièrent d'utiliser des objets de type tableaux. Récemment, Chen et Guo pour les couplages, et Marberg pour les partitions d'ensembles, ont étendu ces résultats au cas de diagrammes arc-annotés coloriés. Nous démontrons que la propriété d'équidistribution s'observe est aussi vraie dans le cas de permutations aux arcs coloriés. Tout comme dans le travail résent de Marberg, mais via un autre chemin, nous montrons que les séries génératrices ordinaires des permutations $r$-coloriées ayant au plus $j$ chevauchements et $k$ emboitements, comptées selon la taille $n$, sont des fonctions rationnelles. Nous décrivons aussi des algorithmes permettant de calculer ces fonctions rationnelles pour les partitions d'ensembles et les permutations coloriées sans emboitement ou sans chevauchement.
\end{abstract}

Keywords: arc-coloured permutation, crossing, nesting, bijection, enumeration, tableau, generating tree, finite state automaton, transfer matrix, automation

\section{Introduction}

Crossing and nesting statistics have intrigued combinatorialists for many decades. For example, it is well known that Catalan numbers, $c_{n}=\frac{1}{n+1}\left(\begin{array}{c}2 n \\ n\end{array}\right)$, count the number of noncrossing matchings on $[2 n]$ which is also the number of nonnesting matchings of the same size. The concept of crossing and nesting 


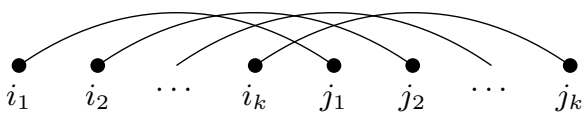

Figure 1: The arc diagram of a $k$-crossing

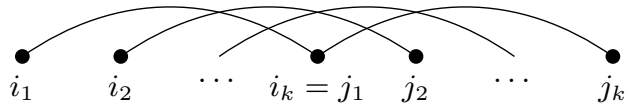

Figure 3: The arc diagram of an enhanced $k$ crossing

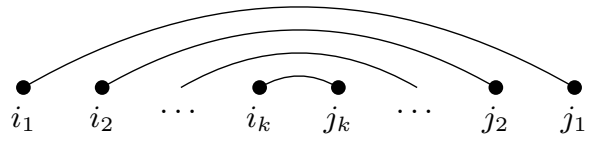

Figure 2: The arc diagram of a $k$-nesting

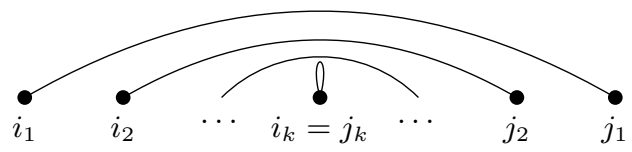

Figure 4: The arc diagram of an enhanced $k$-nesting

was then extended to higher numbers where symmetric joint distribution continues to hold not only for matchings (8), but also for set partitions (4, 9), labelled graphs (7), set partitions of classical types (13), and permutations (2). In all cases, bijective proofs were given; and for some, generating functions were found.

Inspired by recent works of Chen and Guo (3) on coloured matchings and Marberg (10) on coloured set partitions, we give a bijection to establish symmetric joint distribution of crossing and nesting statistics for arc-coloured permutations. We also show that the ordinary generating functions for $j$-noncrossing, $k$-nonnesting, $r$-coloured permutations according to size $n$ are rational functions.

\subsection{Definitions and Terminology}

A permutation $S$ of the set $[n]:=\{1,2, \ldots, n\}$ is a bijection from $[n]$ to itself, $\sigma:[n] \rightarrow[n]$. Using two-line notation, we can write $S=\left(\begin{array}{ccccc}1 & 2 & 3 & \cdots & n \\ \sigma(1) & \sigma(2) & \sigma(3) & \cdots & \sigma(n)\end{array}\right)$. An arc annotated diagram is a labelled graph on $n$ vertices drawn horizontally, labelled left to right consecutively such that $\operatorname{Arc}(i, j)$ joins vertex $i$ to vertex $j$. A permutation has a representation as an arc annotated diagram where $\operatorname{Arc}(i, \sigma(i))$ is drawn as an upper arc for $\sigma(i) \geq i$, and a lower arc for $\sigma(i)<i$. Note that the dissymmetry draws a fixed point in $S$ as an upper loop. When this diagram is restricted to only the upper arcs (or lower arcs) with all $n$ vertices, then it also represents a set partition of $[n]$. Separately, we call these upper and lower arc diagrams of a permutation. From this diagram, we define a $k$-crossing (resp. $k$-nesting) as $k$ arcs $\left\{\left(i_{1}, j_{1}\right),\left(i_{2}, j_{2}\right), \ldots,\left(i_{k}, j_{k}\right)\right\}$ all mutually cross, or $i_{1}<i_{2}<\cdots<i_{k}<j_{1}<j_{2}<\cdots<j_{k}$ (resp. nest, i. e. $i_{1}<i_{2}<\cdots<i_{k}<j_{k}<j_{k-1}<\cdots<j_{1}$ ) as shown in Figure 1 (resp. Figure 2). We also need a variant: enhanced $k$-crossing (resp. enhanced $k$-nesting) where $i_{1}<i_{2}<\cdots<i_{k} \leq j_{1}<j_{2}<$ $\cdots<j_{k}$ (resp. $i_{1}<i_{2}<\cdots<i_{k} \leq j_{k}<j_{k-1}<\cdots<j_{1}$ ) as shown in Figure 3 (resp. Figure 44.

We need both notions of crossings and nestings for permutations because the enhanced definitions are used for upper arc diagrams whereas the other definitions (without enhanced), for lower arc diagrams. This is in accordance with the literature (6) on permutation statistics for weak exceedances and pattern avoidance. We define the crossing number, $\operatorname{cr}(S)=j$ (resp. nesting number, $\operatorname{ne}(S)=k$ ) of a permutation $S$ as the maximum $j$ (resp. $k$ ) such that $S$ has a $j$-enhanced crossing (resp. $k$-enhanced nesting) in the upper arc diagram or a $j$-crossing (resp. $k$-nesting) in the lower arc diagram. When a permutation $S$ does not have a $j$-(enhanced)-crossing (resp. $k$-(enhanced)-nesting), then we say $S$ is $j$-noncrossing (resp. $k$ nonnesting). Burrill, Mishna, and Post (2) gave an involution mapping between the set of permutations of 
$[n]$ with $\operatorname{cr}(S)=j$ and ne $(S)=k$ and those with $\operatorname{cr}(S)=k$ and ne $(S)=j$, thus extending the result of symmetric joint distribution for matchings and set partitions of Chen, Deng, Du, Stanley, and Yan (4) and Krattenthaler (9) to permutations.

Next, Chen and Guo (3) generalized symmetric equidistribution of crossing and nesting statistics to coloured complete matchings. Most recently, Marberg (10) extended the result to coloured set partitions with a novel way of proving that the ordinary generating functions of $j$-noncrossing, $k$-nonnesting, $r$ coloured partitions according to size $n$ are rational functions. We extend their results to $r$-arc-coloured permutations, or $r$-coloured permutations in short.

Coloured permutations are generalizations of permutations represented as arc annotated diagrams. Once the arcs are coloured to satisfy $j$-noncrossing and $k$-nonnesting conditions for each colour class, the resulting arc diagrams can be represented in the topological graph theoretic book embedding setting (11), each colour on a separate page while the vertices are on the spine of the book. The differences are two fold: each page satisfies the crossing/nesting conditions instead of finding a minimum number of pages, noncrossing on each page, to represent a given (non-planar) graph, and the number of pages is not necessarily minimal with respect to the crossing/nesting conditions. Secondary RNA structures with different bonding energies have been analysed in the book embedding setting, naturally represented as coloured set partitions (5); however, arc-coloured permutations have yet to find a natural application.

Some caution on terminology is in order here. Group properties of coloured permutations have been widely studied since the 1990's (1, 15), but there the colours are assigned to vertices instead of arcs.

\subsection{Main Theorem}

Since crossing and nesting statistics involves arcs, we define an $r$-coloured permutation parallel to (10) as a pair, $(S, \phi)$ consisting of a permutation of $[n]$ and an arc-colour assigning map $\phi: \operatorname{Arc}(S) \rightarrow[r]$, and use a capital Greek letter, $\Sigma$, to denote these objects. We say $\Sigma$ has a $k$-crossing (resp. $k$-nesting) if $k$ arcs of the same colour cross (resp. nest). As always throughout this paper, enhanced statistics is applied to upper arc diagrams while non-enhanced for lower arc diagrams of permutations. Define $\operatorname{cr}(\Sigma)$ (resp. ne $(\Sigma)$ ) as the maximum integer $k$ such that $\Sigma$ has a $k$-crossing (resp. $k$-nesting). The bijection of (2) can be extended to establish symmetric joint distribution of the numbers $\operatorname{cr}(\Sigma)$ and ne $(\Sigma)$ over $r$-coloured permutations preserving opener and closer sequences (equivalently, sets of minimal and maximal elements of each block when upper arc and lower arc diagrams are viewed separately as set partitions).

More formally, vertices of a permutation are of five types, an opener $(\mathcal{C})$, a closer ( $)$, a fixed point ( $\bullet$ ), an upper transitory ( $\checkmark$ ), and a lower transitory ( $)$. For a particular $\Sigma$, restricting to only one colour, both upper arc and lower arc diagrams can be seen as set partitions whose minimal block elements are the openers, and maximal block elements are the closers. For upper arc diagrams, both a fixed point and an upper transitory contribute to the set of minimal (opener) and the set of maximal (closer) elements over blocks of the set partition. Lower arc diagrams are set partitions in Marberg's partition setting, thus Theorem 1.1 and Corollary 1.2 of (10) apply exactly here.

Given an $r$-coloured permutation $\Sigma=(S, \phi)$, let the set of openers (resp. the set of closers) be $\mathcal{O}(\Sigma)$ (resp. $\mathcal{C}(\Sigma)$ ) of the uncoloured permutation, $S$. For all positive integers, $j$ and $k$, and subsets $O, C \subseteq[n]$, define $\mathrm{NCN}_{j, k}^{O, C}(n, r)$ to be the number of $r$-coloured permutations $\Sigma$ of $[n]$ with $\operatorname{cr}(\Sigma)<j$, ne $(\Sigma)<k$, $\mathcal{O}(\Sigma)=O$, and $\mathcal{C}(\Sigma)=C$. Then Theorem 1 is analogous to Theorem 1.1 in (4, 10) for $r$-coloured permutations. 
Theorem 1 For all positive integers, $j$ and $k$, and subsets $O, C \subseteq[n], \operatorname{NCN}_{j, k}^{O, C}(n, r)=\operatorname{NCN}_{k, j}^{O, C}(n, r)$.

As customary in the literature, we let $\mathrm{NCN}_{j, k}(n, r)$ denote the number of all $r$-coloured, $j$-noncrossing, $k$-nonnesting permutations of $[n]$. Summing both sides of Theorem 1 over all $O, C \subseteq[n]$ gives the generalization of (4, 10) for Corollary 1 . We also let $\mathrm{NC}_{k}(n, r)$ (resp. $\mathrm{NN}_{k}(n, r)$ ) denote the number of $k$-noncrossing (resp. $k$-nonnesting) $r$-coloured permutations on $[n]$.

Corollary 1 For all integers, $j, k, n, r, \mathrm{NCN}_{j, k}(n, r)=\mathrm{NCN}_{k, j}(n, r)$ and $\mathrm{NC}_{k}(n, r)=\mathrm{NN}_{k}(n, r)$.

\subsection{Plan}

The tools needed for the proof of Theorem 1 are given in Section 2. Section 3 gives the proof of Theorem 1 combining essential ingredients of both $(2,10)$ with the added care of managing both upper and lower arc diagrams simultaneously where both notions of crossing and nesting are applied. The transfer matrix approach Marberg used to establish the rationality of the ordinary generating function, $\sum_{n \geq 0} \mathrm{NCN}_{j, k}(n+$ $1, r) x^{n}$ for set partitions of size $n+1$ is through translating the original problem to counting all closed walks of $n$-steps with certain column and row length restrictions (according to $j, k$ ) for each component from $\emptyset \in \mathbf{Y}^{r}$, that is, $r$ copies of the Hasse diagram of the Young lattice. This idea cannot be extended to permutations on $\left(\mathbf{Y}^{r}, \mathbf{Y}^{r}\right)$ because upper arc diagrams are dependent on lower arc diagrams. However, another interpretation of Marberg's multigraphs $\mathcal{G}_{j, k, r}$ in terms of the types of vertices and colours of edges leads to the multigraphs for $r$-coloured permutations which permits the application of transfer matrix method to draw the same conclusion: The ordinary generating function, $\sum_{n>0} \mathrm{NCN}_{j, k}(n, r) x^{n}$ for $j$-noncrossing, $k$-nonnesting, $r$-coloured permutations is rational. The combination of the method of generating trees and finite state automata in the interpretation can be extended to other combinatorial objects where both crossing and nesting statistics are bounded, thus leading to the same conclusion that the corresponding generating functions are rational.

\section{Background}

The proof of Theorem 1 requires working knowledge of the theory of integer partition, especially its representation as Young diagrams, the Hasse diagram of the Young lattice, and the RSK-algorithm for filling positive integers to obtain the beginning of some standard Young tableau. We refer the reader to Volume 2 of Stanley's Enumerative Combinatorics (14) for more details.

Define a partition of $n \in \mathbf{N}$ to be a sequence $\lambda=\left(\lambda_{1}, \lambda_{2}, \ldots, \lambda_{k}\right) \in \mathbf{N}^{k}$ such that $\sum_{i=1}^{k} \lambda_{i}=n$, and $\lambda_{1} \geq \lambda_{2} \geq \cdots \geq \lambda_{k}$. If $\lambda$ is a partition of $n$, we write $\lambda \vdash n$ or $|\lambda|=n$. The non-zero terms $\lambda_{i}$ are called the parts of $\lambda$, and we say $\lambda$ has $k$ parts if $\lambda_{k}>0$. We can draw $\lambda$ using a left-justified array of boxes with $\lambda_{i}$ boxes in row $i$. For example, $\lambda=(5,3,2,2,1)$ is drawn as . This representation is the Young diagram of a partition. To "add a box" to a partition $\lambda$ means to obtain a partition $\mu$ such that $|\lambda|+1=|\mu|$, and $\lambda$ 's Young diagram is included in that of $\mu$. This inclusion induces a partial order on the set of partitions of non-negative integers, denoted by $\mathbf{Y}$, or the Young lattice. When we place integers $1,2, \ldots, n$ in all $n$ boxes of a Young diagram so that entries increase in each row and column, we produce a standard Young tableau, abbreviated as SYT. As one builds an SYT from the empty set through the process of adding a box at a time, a sequence of integer partitions, $\left(\lambda^{0}=\emptyset, \lambda^{1}, \lambda^{2}, \ldots, \lambda^{n}\right)$ emerges where $\lambda^{i-1} \subset \lambda^{i}$, and $\left|\lambda^{i}\right|=\left|\lambda^{i-1}\right|+1$. In addition to adding a box, we include "deleting a box" and "doing nothing" for the following four types in Definition 1 . 
Definition 1 We define four types of sequences of tableaux, $T=\left(\lambda^{0}=\emptyset, \lambda^{1}, \lambda^{2}, \ldots, \lambda^{n}\right)$, where $\lambda^{0}=$ $\lambda^{n}=\emptyset$ such that $\lambda^{i}$ is obtained from $\lambda^{i-1}$ for each $i \in[n]$ by one of the three actions: adding a box, deleting a box, or doing nothing.

1. A semi-oscillating tableau is any such sequence $T$.

2. An oscillating tableau has distinct neighbouring $\lambda^{i}$ 's.

3. A vacillating tableau is any such sequence $T$ which has $\lambda^{i-1} \subseteq \lambda^{i}$ when $i$ is even, and $\lambda^{i-1} \supseteq \lambda^{i}$ when $i$ is odd.

4. A hesitating tableau is any such sequence $T$ which has $\lambda^{i-1} \subseteq \lambda^{i}$ when $i$ is odd, and $\lambda^{i-1} \supseteq \lambda^{i}$ when $i$ is even.

In the uncoloured case, Marberg (10) links the sequence $T$ to an $n$-step walk on the Hasse diagram of the Young lattice, $\mathbf{Y}$ where "doing nothing" is also counted as a step. For his enumeration purposes, Marberg's definitions differ slightly from (4) to achieve that these $n$-step walks are closed walks from $\emptyset$. Though we will not walk on an ordered pair of $r$-tuple Hasse diagrams, we will keep the requirement that each sequence $T$ begins and ends with $\emptyset$.

\section{Proof of Main Theorem}

The proof of Theorem 1 needs two local rules for changing set partitions to involutions: Rule $\mathrm{H}$ for hesitating tableaux tracking enhanced statistics in upper arcs and Rule V for vacillating tableaux.

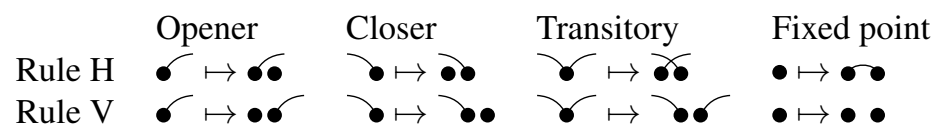

\subsection{Proof of Theorem 1}

Proof: We show an involution between the set of $r$-coloured permutations of $[n]$ with maximal crossing number $j$, nesting number $k$ and those with maximal crossing number $k$ and nesting number $j$.

Given an $r$-coloured permutation of $[n]$, say $\Sigma=(S, \phi)$, first consider its corresponding uncoloured permutation $S$. Let $O$ be $\mathcal{O}(S)$, the set of openers and $C$ be $\mathcal{C}(S)$, the set of closers. Applying the involution of (2) results in another permutation with the same $O$ and $C$ while switching maximal crossing and nesting numbers.

Now for each colour class, the resulting arc diagram is no longer a permutation, but two set partitions: enhanced for the upper arc diagram, and non-enhanced for the lower arc diagram. We employ the same encoding techniques from (2):

Step 1 Translate the upper arc diagram into a hesitating tableau sequence, and the lower arc diagram into a vacillating tableau sequence.

Step 2 Perform a component-wise transpose to each tableau sequence.

Step 3 Apply reverse RSK to fill each tableau in the sequence from the right to the left.

Step 4 Translate the newly filled sequence of tableaux back to arc diagrams according to its own rule. 
Thus, we obtain the resulting arc diagram with its upper and lower arc components where maximal crossing and nesting numbers are switched because the bijections of $(2,4,9)$ interchange maximal column length with maximal row length while preserving sets of maximal and minimal block elements. This interchange achieved through taking the conjugate (transpose) of each tableau translates to the switching of maximal nesting and crossing numbers while preserving the sets of openers and closers. The preservation of these sets when restricted to one colour of arcs permits the involution to be applied separately to all arcs of the same colour, one colour at a time, without interfering with the sets of openers and closers from other colour classes. Finally, the combination of all $r$ involutions, one for each colour, produces the desired $r$-coloured permutation such that for each colour, crossing number and nesting number are switched. If the original $r$-coloured $\Sigma$ is $j$-noncrossing and $k$-nonnesting, then its image after the $r$-fold involution is $j$-nonnesting and $k$-noncrossing.

\subsection{An example of a 2-coloured permutation}

We show a 2-coloured permutation where we apply the involution of the proof of Theorem 1 to find its image.

Example 1 A permutation encoded by a hesitating tableau sequence, $\lambda_{1}$ for colour $1, \lambda_{2}$ for colour 2 in the upper arcs and $a$ vacillating tableau sequence, $\mu_{2}$ for colour 2 in the lower arcs.
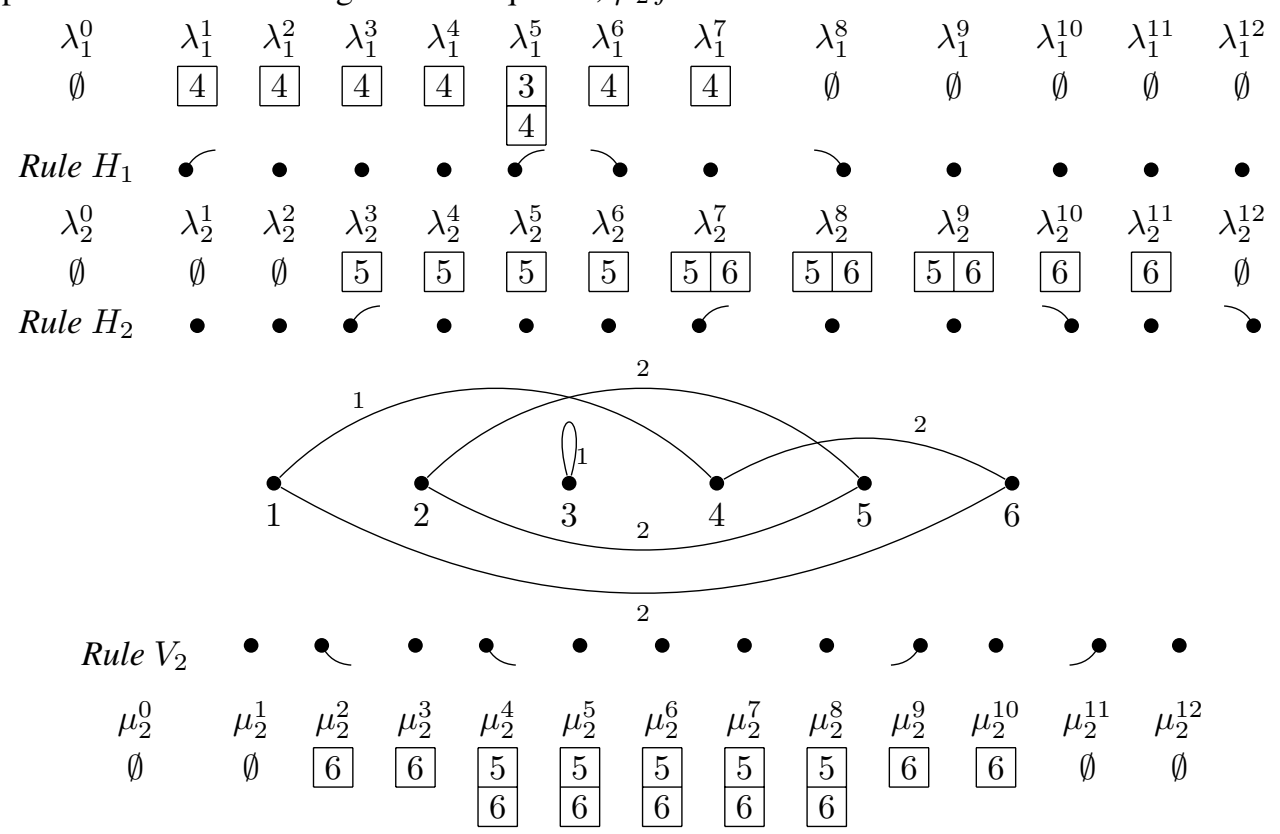

The result of transposing every tableau in each sequence $\lambda_{1}, \lambda_{2}$, and $\mu_{2}$, and filling the tableau from the right is the following 2-coloured permutation in Figure 5 .

\section{Enumeration of $r$-coloured permutations}

Before we enumerate $r$-coloured permutations, a quick overview of Marberg's approach for the enumeration of coloured set partitions helps set the stage for a new interpretation. 


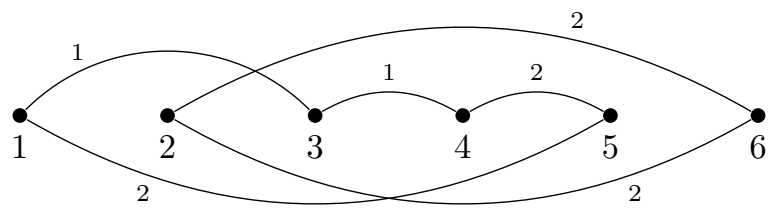

Figure 5: The image of Example 1 under the involution in the proof of Theorem 1

\subsection{Another interpretation of $\mathcal{G}_{j, k, r}$ for set partitions}

Marberg viewed $r$ sequences of vacillating tableaux, one for each colour, as $r \times(k-1)$ matrices $A=\left[A_{i, l}\right]$ encoding $\lambda_{i}^{l}$ in a vacillating tableau sequence $T$ for colour $i$. If the set partition is $j$-noncrossing and $k$-nonnesting, then this tableau has a maximum of $j-1$ columns and $k-1$ rows. For colour $i$, the $i$ th row of matrix $A$ just lists parts of $\lambda^{l}$, thus at most $k-1$ non-zero parts. The multigraph $\mathcal{G}_{j, k, r}$ is drawn using all such allowable $A$ 's as vertices, and edges and loops connecting vertices corresponding to adding a box, deleting a box, or doing nothing in the construction of vacillating tableaux so that the resulting sequence contains only tableaux of at most $j-1$ columns and $k-1$ rows. Once completed, the multigraph $\mathcal{G}_{j, k, r}$ gives rise to an adjacency matrix. To find the number $\operatorname{NCN}_{j, k}(n, r)$ which is also the number of $(n-1)$-step walks on $\mathcal{G}_{j, k, r}$ from the zero matrix to itself, the method of transfer matrix gives a quotient of two polynomials (determinants actually), thus concluding that the ordinary generating function $\sum_{n \geq 0} \mathrm{NCN}_{j, k}(n+1, r) x^{n}$ is rational.

\subsection{Examples of $\mathcal{G}_{2,2,1}$ and $\mathcal{G}_{2,2,2}$ for set partitions}

To illustrate the construction of $\mathcal{G}_{j, k, r}$, we first reconstruct Marberg's $\mathcal{G}_{2,2,1}$ and $\mathcal{G}_{2,2,2}$ by naming each vertex and edge as it becomes necessary.

The arc annotated diagram of a set partition on $[n]$ has $n-1$ consecutive gaps, i. e. between each pair of adjacent points. Let the set of non-crossing, non-nesting, uncoloured set partitions on $[n]$ be denoted by $\mathcal{P}_{2,2,1}(n)$. For each $P \in \mathcal{P}_{2,2,1}(n)$, a snap shot of each gap belongs to one of the first four types in Table 1 where the matching steps in $\mathcal{G}_{2,2,1}$ are also given. Since $r=1$, only two vertices exist in $\mathcal{G}_{2,2,1}$ : $v_{0}$, the initial state for no opener, and $v_{1}$, for one opener. No other vertices accounting for other states are present because any state $v_{i}$ where $i \geq 2$ would mean two or more openers which will form at least a 2-nesting or 2-crossing when closed. Incident at $v_{0}$ are three types of edges: two loops, $\ell^{\times}$for no arc in the consecutive gap, and $b^{1}$ for a distance 1-arc both of which do not change the number of openers present as the set partition is scanned from the left to the right; the last type is a directed edge from $v_{0}$ to $v_{1}$ to indicate that an opener is present in the consecutive gap. Once at $v_{1}$, only the loop, $\ell^{\times}$, is allowed because a 1-arc ${ }^{1}$ will create a 2 -nesting in $P$ with the existing opener. A directed edge from $v_{1}$ to $v_{0}$ means that an opener is closed. To simplify drawing, an edge without arrows is bidirectional. The result is shown in Figure6.

To construct $\mathcal{G}_{2,2,2}$, we require four vertices: still $v_{0}$ as the initial state for no opener, but also two states indicating one $r$-coloured $(r \in[2])$ opener, $v_{1_{1}}$ and $v_{1_{2}}$. Since two arcs of different colours do not create a crossing or nesting, one more state is needed, $v_{2_{12}}$, for two openers, one of each colour. As in $\mathcal{G}_{2,2,1}$, 


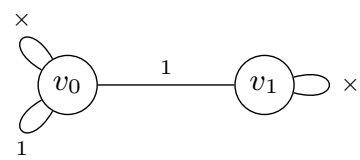

Figure 6: An uncoloured set partition graph, $\mathcal{G}_{2,2,1}$.

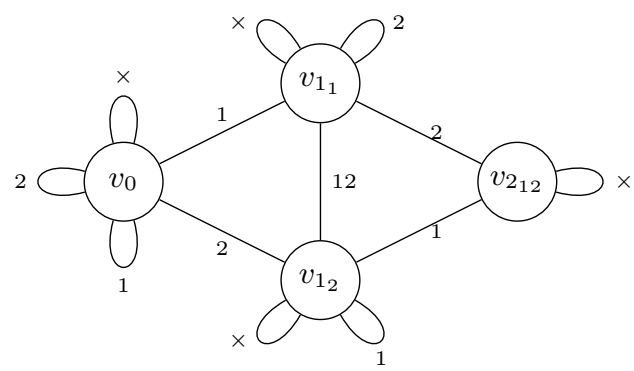

Figure 7: A 2-coloured set partition graph, $\mathcal{G}_{2,2,2}$.

the loops and edges are placed according to what is allowed in $P$, but a new edge between $v_{1_{1}}$ and $v_{1_{2}}$ is added in the last row of Table 1 for the closing of one colour on point $m$ while an opener is present at point $m-1$ in $P$. The result is shown in Figure 7

For details on how the adjacency matrices for Figures 6 and 7 give rise to generating functions, please see (16).

In general, we obtain $\mathcal{G}_{j, k, r}$ directly through labelling the edges and vertices of $\mathcal{G}_{j, k, r}$ similar to generating such set partitions through the method of generating trees except that each vertex $v_{i}$ (considered as a state) in $\mathcal{G}_{j, k, r}$ indicates that $i$ openers are pending to close. When drawn from the left to the right where all vertices of the same first subscript line up vertically, we get edges either between $v_{i}$ and $v_{i+1}$ for each $i \geq 0$ for openers or closers as in Figure 8 , or between vertices of the same first subscript for the presence of both (drawn as vertical edges, not shown in Figure 8). Care needs to be taken when many arcs of the same colour are open because the order in which they are closed relates to how crossing and nesting are formed.

We list the first few series for $\mathcal{G}_{2,2, r}, r=\{3,4\}$. The first two series, $r=1,2$ were found by Marberg (10) where A216949 in (12) is for $r=2$. Our series mark the number of consecutive gaps, namely, $x^{k}$ counts the number of such coloured set partitions on $k+1$ elements. For more terms and the rational functions, please consult A225029-A225033 in (12) for $r=3$ to 7 .

$\sum_{n \geq 0} \mathrm{NCN}_{2,2}(n, 3) x^{n}=\frac{1-10 x+22 x^{2}-x^{3}}{1-14 x+59 x^{2}-74 x^{3}+x^{4}}=1+4 x+19 x^{2}+103 x^{3}+616 x^{4}+3949 x^{5}+\ldots$
$\sum_{n \geq 0} \mathrm{NCN}_{2,2}(n, 4) x^{n}=\frac{1-20 x+122 x^{2}-224 x^{3}+x^{4}}{1-25 x+218 x^{2}-782 x^{3}+973 x^{4}-x^{5}}=1+5 x+29 x^{2}+193 x^{3}+1441 x^{4}+\ldots$

Using an average personal computer, Maple15 can generate up to 7 colours. The next case, $r=8$, with a matrix size of $256 \times 256$, computation would take too long to find the determinants.

\subsection{Multigraphs, $\mathcal{G}_{2,2,1}$ and $\mathcal{G}_{2,2,2}$ for permutations}

Instead of translating consecutive gaps from set partitions into steps in the multigraph $\mathcal{G}$, we examine each vertex in the arc diagram of a coloured permutation and assign each type of vertex to a step in $\mathcal{G}$. As for set partitions, we first construct the multigraph $\mathcal{G}_{2,2,1}$ for non-crossing, non-nesting, uncoloured permutations. Let us denote the set of all such permutations on $[n]$ by $\mathcal{S}_{2,2,1}(n)$. If $S \in \mathcal{S}_{2,2,1}(n)$, then a 


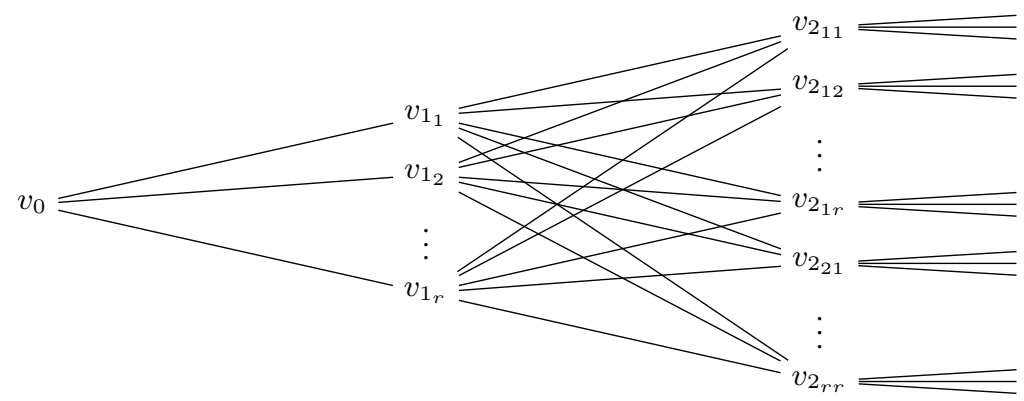

Figure 8: The line-up for states of the same number of openers

\begin{tabular}{|c|c|c|c|}
\hline Domain & Set partition & Types of arcs & Types of steps in $\mathcal{G}_{2,2,1}$ and $\mathcal{G}_{2,2,2}$ \\
\hline$m \geq 2$ & $m-1 \quad \stackrel{\bullet}{\bullet}$ & no arc & $e^{x}$ \\
\hline$m \geq 2$ & & a 1-arc coloured $r$ & , $r \in[2]$ \\
\hline$m \geq 2$ & $m-1 \quad m$ & an opener & -1 \\
\hline$m \geq 3$ & $m-1 \quad \stackrel{\bullet}{\bullet}$ & a closer & $\grave{v}_{i} \quad r \quad \overleftrightarrow{v}_{i+1}^{\bullet}$ \\
\hline$m \geq 3$ & $\overbrace{m-1}^{1}$ & a closer and an opener & $\begin{array}{l}12 \\
v_{i_{2}}\end{array}$ \\
\hline
\end{tabular}

Table 1: Five situations between point $m-1$ and point $m$ for set partitions and the matching steps in $\mathcal{G}$.

vertex is either a fixed point ( $)$, an opener $(\prec)$, a closer ( $\supset$ ), or a lower transitory $(\ell)$ ). We can't have an upper transitory which contributes to a 2 -(enhanced) crossing.

In Figure 9, $v_{0}$ still indicates the initial state with 0 opener; $v_{1}$ indicates the state with 1 opener. The loop labelled 1 is the step taken when a fixed point coloured 1 is encountered in the permutation scanned from the left. The loop labelled $1_{t}$ is the presence of a lower transitory with coloured 1 arcs on both sides; this is possible only when an opener coloured 1 is present, thus at $v_{1}$. Note that a lower transitory does not alter the state. The directed edge $\left(v_{0}, v_{1}\right)$ indicates the presence of an opener, and the edge traversed in reverse indicates that of a closer. An edge drawn without arrows still means a bidirectional edge.

The construction of $\mathcal{G}_{2,2,2}$ involves more types of vertices and edges which we summarize in Table 2

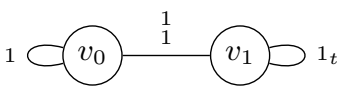

Figure 9: An uncoloured permutation graph, $\mathcal{G}_{2,2,1}$. 
Each state with one opener has the colours of the openers as subscripts. When a state has two openers, both colours are used, thus only one such vertex in $v_{2}$. The method of transfer matrix gives the following generating function. Here $x$ marks the size of the permutation.

$\sum_{n \geq 0} \mathrm{NCN}_{2,2}(n, 2) x^{n}=\frac{1-6 x+4 x^{2}}{(1-2 x)(1-6 x)}=1+2 x+8 x^{2}+40 x^{3}+224 x^{4}+1312 x^{5}+7808 x^{6}+O\left(x^{7}\right)$.

This series, A092807 in (12), counts (with interpolated zeros) the number of closed walks of length $n$ at a vertex of the edge-vertex incidence graph of $K_{4}$, the complete graph on 4 vertices associated with the edges of $K_{4}$. The next two series, A224992 and A224993 in (12), however, are new. For 5 colours, the matrix size, $252 \times 252$, hinders fast computation of determinants.

$$
\begin{gathered}
\sum_{n \geq 0} \mathrm{NCN}_{2,2}(n, 3) x^{n}=\frac{1-17 x+66 x^{2}-36 x^{3}}{(1-2 x)(1-6 x)(1-12 x)}=1+3 x+18 x^{2}+144 x^{3}+1368 x^{4}+O\left(x^{5}\right), \\
\sum_{n \geq 0} \mathrm{NCN}_{2,2}(n, 4) x^{n}=\frac{1-36 x+380 x^{2}-1200 x^{3}+576 x^{4}}{(1-2 x)(1-6 x)(1-12 x)(1-20 x)}=1+4 x+32 x^{2}+352 x^{3}+4736 x^{4}+O\left(x^{5}\right) .
\end{gathered}
$$

\begin{tabular}{|c|c|c|c|}
\hline Domain & Permutation Vertex & Types of arcs & Types of steps in $\mathcal{G}_{2,2,2}$ \\
\hline all vertices & 8 & a fixed point & $\ell^{l}, l \in[2]$ \\
\hline all except the last & & an opener & $\vec{v}_{i+1}^{\bullet}, r, s \in[2]$ \\
\hline $\begin{array}{l}\text { all except the first } \\
\text { no first, no last }\end{array}$ & & $\begin{array}{c}\text { a closer } \\
\text { a lower transitory }\end{array}$ & $\begin{array}{c}v_{i} \quad v_{i+1}, r, s \in \\
\oint^{r_{t}}, r \in[2]\end{array}$ \\
\hline no first, no last & & an upper transitory & - $v_{i_{s}}, r, s \in[2]$ \\
\hline no first, no last & s & a lower transitory & - $v_{i_{s}}, r, s \in[2]$ \\
\hline
\end{tabular}

Table 2: Vertices in permutations and the matching steps in $\mathcal{G}_{2,2,2}$.

\subsection{Proof of Rationality through Multigraphs for $r$-coloured permutations}

In general, drawing $\mathcal{G}_{j, k, r}$ for coloured permutations is a tedious task. As the $j, k$, and $r$ increase, types of edges and vertices increase. Not only does one need to track the order in which coloured arcs are closed, one also needs to create unidirectional edges which go to the right states. Regardless of the complexity of 


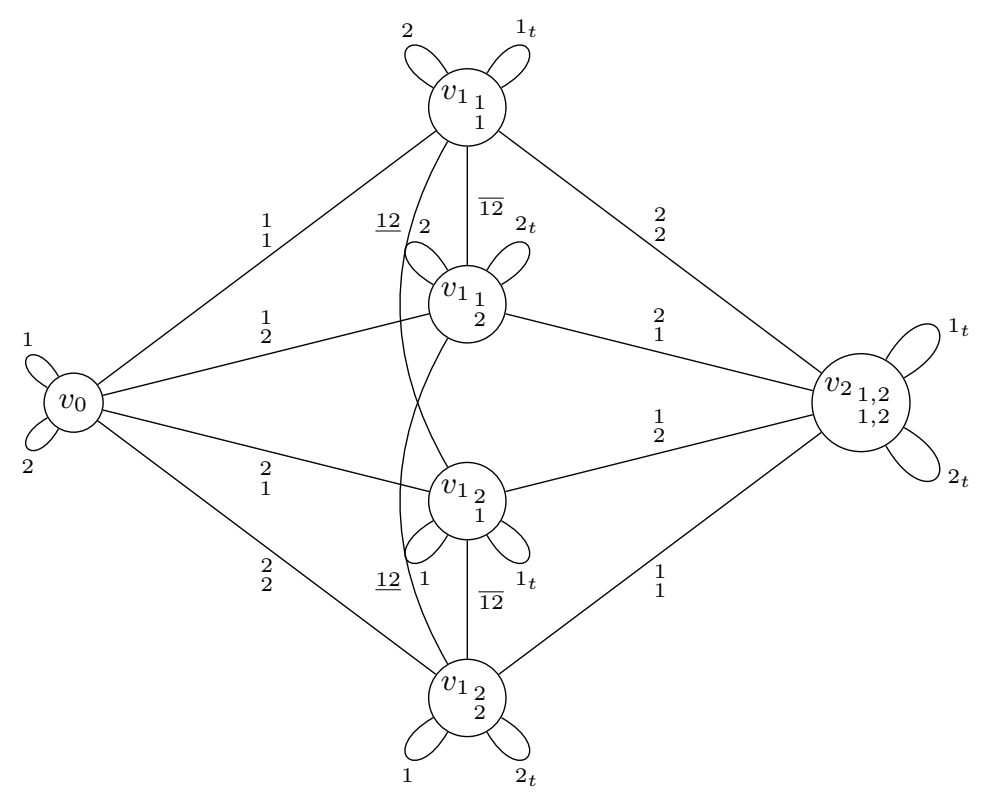

Figure 10: A 2-coloured permutation multigraph, $\mathcal{G}_{2,2,2}$

the multigraph, $\mathcal{G}_{j, k, r}$, only a finite number of vertices and edges are present because both crossing and nesting numbers are bounded for the set of $r$-coloured permutations. Furthermore, the number of such permutations on $[n]$ is the number of $n$-step paths from $v_{0}$ to $v_{0}$ in $\mathcal{G}_{j, k, r}$ because all openers must be closed. Using the method of transfer matrix then yields a rational function for the ordinary generating function, $\sum_{n \geq 0} \mathrm{NCN}_{j, k}(n, r) x^{n}$.

\section{Concluding Remarks}

When both nesting and crossing numbers are bounded, a finite multigraph can be constructed. This method of transfer matrix may be extended to the enumeration of set partitions of classical types as in the works of Rubey and Stump (13), even their coloured counterparts. The challenge lies in finding the generating function when only one of the bounds is present. For instance, Marberg (10) showed that the ordinary generating function for noncrossing 2-coloured set partitions is D-finite, but conjectured non-Dfinite series for noncrossing $r$-coloured set partitions when $r \geq 3$.

\section{Acknowledgements}

The author would like to thank Marni Mishna for recommending Marberg's paper (10), Eric Marberg for clarifying the construction of the matrices which act as vertex labels of the multigraph $\mathcal{G}_{j, k, r}$, and two anonymous referees for their constructive comments which led to the automation of generating the rational series for coloured noncrossing nonnesting set partitions and permutations. Mogens Lemvig Hansen helped with figures and Maple code (16) for the automation. 


\section{References}

[1] Alexei Borodin. Longest increasing subsequences of random colored permutations. Electron. J. Combin., 6(1):R13, 12 pp. (electronic), 1999. ISSN 1077-8926.

[2] Sophie Burrill, Marni Mishna, and Jacob Post. On $k$-crossings and $k$-nestings of permutations. In Proceedings of 22nd International Conference on Formal Power Series and Algebraic Combinatorics, pages 593-600, San Francisco, CA, USA, 2010.

[3] William Y. C. Chen and P. L. Guo. Oscillating rim hook tableaux and colored matchings. $A d v$. in Appl. Math., 48(2):393-406, 2011.

[4] William Y. C. Chen, Eva Y. P. Deng, Rosena R. X. Du, Richard P. Stanley, and Catherine H. Yan. Crossings and nestings of matchings and partitions. Trans. Amer. Math. Soc., 359(4):1555-1575 (electronic), 2007. ISSN 0002-9947.

[5] Peter Clote, Stefan Dobrev, Ivan Dotu, Evangelos Kranakis, Danny Krizanc, and Jorge Urrutia. On the page number of RNA secondary structures with pseudoknots. J. Math. Biol., 65(6-7):1337-1357, 2012.

[6] Sylvie Corteel. Crossings and alignments of permutations. Adv. in Appl. Math., 38(2):149-163, 2007. ISSN 0196-8858.

[7] Anna de Mier. $k$-noncrossing and $k$-nonnesting graphs and fillings of Ferrers diagrams. Combinatorica, 27(6):699-720, 2007. ISSN 0209-9683.

[8] Dominique Gouyou-Beauchamps. Standard Young tableaux of height 4 and 5. European Journal of Combinatorics, 10:69-82, 1989.

[9] Christian Krattenthaler. Growth diagrams, and increasing and decreasing chains in fillings of Ferrers shapes. Adv. in Appl. Math., 37(3):404-431, 2006. ISSN 0196-8858.

[10] Eric Marberg. Crossings and nestings in colored set partitions. preprint, October 2012. URL http://arxiv.org/abs/1203.5738, Last accessed April 2013.

[11] Miki Miyauchi. Topological book embedding of bipartite graphs. IEICE TRANSACTIONS, E89-A (5):1223-1226, 2006. ISSN 0916-8508. doi: http://dx.doi.org/10.1093/ietfec/e89-a.5.1223.

[12] OEIS Foundation Inc. The On-Line Encyclopedia of Integer Sequences. Published electronically at http://oeis.org, 2011.

[13] Martin Rubey and Christian Stump. Crossings and nestings in set partitions of classical types. Electr. J. Comb., 17(1):Reserach Paper 120, 22 pp. (electronic), 2010.

[14] Richard P. Stanley. Enumerative Combinatorics, volume 2. Cambridge University Press, 1999.

[15] Christian Stump. More bijective Catalan combinatorics on permutations and on colored permutations. preprint, June 2012. URL http://arxiv.org/abs/0808.2822 Last accessed November 2012.

[16] Lily Yen. Crossings and nestings for arc-coloured permutations. Preprint, submitted, November 2012. URL http://arxiv.org/abs/1211.3472 